\title{
ON POINTS OF EXTREME CHAOS FOR ALMOST CONTINUOUS FUNCTIONS
}

\author{
RYSZARD J. PAWLAK
}

\begin{abstract}
In this paper, we introduce a new notion: extremely chaotic point and, moreover, a dual notion: family of functions having extremely chaotic point property. The basic properties of such a kind of families of functions will be considered.
\end{abstract}

\section{Introduction and preliminaries}

It is well-known that there exists a number of different (nonequivalent) definitions of chaos. But one can link the main idea of this notion with entropy. This is signaled, for example, by the following quotation from the paper ([4, p. 80]): It is commonly accepted that an evidence of chaos is positivity of topological entropy. Therefore, we say that the dynamical system $(X, f)$ is topologically chaotic if it fulfills the condition $h(f)>0$. It is well-known that topological entropy is closely connected with measure entropy and, consequently, with the entropy of partition. With regard to the last notion, Z. H. Nitecki ([8, p. 9]) states: ... noted that the "entropy" of the partition [...] measures the a priori uncertainty the outcome of an observation-or conversely the information we obtain from performing the observation. This means that, from the topological point of view, the main idea of chaos is connected with the unpredictability of the behaviour of function which, in the language of entropy, can be expressed in the stronger version: topological entropy of function is infinite.

On the other hand, one can observe that sometimes entropy is focused around some point. This fact has become a cause of distinguishing so called entropy points and finally $\infty$-entropy points $[3$. In particular, one can say that $\infty$-entropy point is a chaotic point. But more interesting is the more complicated case when

(c) 2015 Mathematical Institute, Slovak Academy of Sciences.

2010 Mathematics Subject Classification: 26A18, 37B40, 26A21, 54H25, 54C08, $54 \mathrm{H} 20$.

Keywords: topological entropy, almost continuity, extremely chaotic point, family having extremely chaotic point property, $\infty$-entropy point, pseudo fixed point, topology of uniform convergence, $\Gamma$-topology, bitopological space. 
arbitrarily close to the point there are ("in the same proportion") $\infty$-entropy points (unpredictable behaviour of the function), as well as points of entropy 0 (completely predictable function). What is more, we will demand that the set of the points of $\infty$-entropy is a type "black hole", i.e., each point belonging to this set is also an $\infty$-entropy point for functions lying sufficiently close to our function. Such kind of points will be called extremely chaotic points of a function (Definition 2.1).

Now, let us establish some notations and notions to be used in this paper.

We will consider exclusively self-maps $f:[0,1] \rightarrow[0,1]$ which are almost continuous functions. This kind of functions has been introduced by J. St alling $\mathrm{s}$ in 13 in order to consider possible wide class of functions having fixed point property (see Lemma 2.5). Since considerations regarding entropy points are also often connected with fixed points, so limiting consideration to the almost continuous functions seems to be justified. As in a number of papers connected with almost continuity, no distinction is made between a function and its graph (it should be clear from the context).

A function $f$ is almost continuous (in the sense of Stallings) if each nonempty open set $U \subset[0,1]^{2}$ containing $f$ contains also some continuous function defined on $[0,1]$. The set of all almost continuous functions $f:[0,1] \rightarrow[0,1]$ will be denoted by $\mathcal{A}$.

In other words, an almost continuous function is one whose graph can be approximated by graphs of continuous functions.

Almost continuous functions have been widely studied by many mathematicians, because they have many interesting properties (from the point of view of real analysis as well as of topology). For example, in our case each, almost continuous function $f$ is a connectivity function (i.e., graph of $f$ restricted to an arbitrary connected set is connected), and so, $f$ is a Darboux function [13. Many properties of these functions became part of the so-called "mathematical folklore". Two papers [6] and [7] are particularly important in this issue. So, if we will apply results contained in these papers, we will only signal this fact by means of citations. For the sake of convenience, we will formulate other results, used in the proofs, as lemmas (certainly, for known results we will signal suitable citations).

An element $\zeta$ is a left-hand (right-hand) cluster number of $f$ at $x_{0}$ with respect to a set $A \subset[0,1]$ if there exists an increasing sequence $\left\{x_{n}\right\}_{n \in \mathbb{N}} \subset A$ (a decreasing sequence $\left.\left\{y_{n}\right\}_{n \in \mathbb{N}} \subset A\right)$ tending to $x_{0}$ such that $\lim _{n \rightarrow \infty} f\left(x_{n}\right)=\zeta$ $\left(\lim _{n \rightarrow \infty} f\left(y_{n}\right)=\zeta\right)$. The set of all left-hand (right-hand) cluster numbers of $f$ at $x_{0}$ with respect to $A$ will be denoted by $L_{A}^{-}\left(f, x_{0}\right)\left(L_{A}^{+}\left(f, x_{0}\right)\right)$. Moreover, we put $L_{A}\left(f, x_{0}\right)=L_{A}^{-}\left(f, x_{0}\right) \cup L_{A}^{+}\left(f, x_{0}\right)$. If $A=[0,1]$, then the subscript $A$ will be omitted. 
The Euclidean metric in $[0,1]^{2}$ will be denoted by $\varrho$. Following the papers [5], [10, [12, we may equip the family of functions $\mathcal{A}$ with two topologies: topology $\mathcal{T}_{u}$ generated by the metric of uniform convergence

$$
\varrho_{u}(f, g)=\sup _{x \in[0,1]}|f(x)-g(x)|
$$

and $\Gamma$-topology (or graph topology)

$$
\mathcal{T}_{\Gamma}=\left\{\{g \in \mathcal{A}: g \in U\}: U \in \mathcal{T}_{2}\right\},
$$

where $\mathcal{T}_{2}$ denotes the Euclidean topology in $[0,1]^{2}$. According to this definition and earlier agreement, the notation $U \in \mathcal{T}_{\Gamma}$ will mean that $U$ is an open set both in $[0,1]^{2}$ as well as in topological space $\left(\mathcal{A}, \mathcal{T}_{\Gamma}\right)$, the distinction of both cases will be clear from the context. $B_{u}(f, \varepsilon)$ will denote an open ball in $\left(\mathcal{A}, \varrho_{u}\right)$ with the centre at the function $f$ and radius $\varepsilon>0$.

Considering the two topologies in $\mathcal{A}$, we naturally come to the issues related to bitopological space $([2])\left(\mathcal{A}, \mathcal{T}_{\Gamma}, \mathcal{T}_{u}\right)$. In Theorem 3.4 , we will use the notion of a nowhere dense set in bitopological space. A detailed analysis of the different concepts of this notion is not the aim of this paper. Taking advantage of the specificity of our topology, slightly modifying Definition 2.3 contained in [9], we assume that a set $A$ is a $\left(\mathcal{T}_{\Gamma}, \mathcal{T}_{u}\right)$-nowhere dense if for each nonempty open set $W \in \mathcal{T}_{\Gamma}$ there is a nonempty set $V \in \mathcal{T}_{u}$ such that $V \subset W$ and $V \cap A=\emptyset$.

We will use standard topological denotations. The letter $\mathbb{N}$ will denote the set of all positive integers. If $A \subset[0,1]$, then $\operatorname{int} A, \bar{A}$, $\operatorname{dia}(A)$ denote the interior, the closure and the diameter of a set $A$, respectively. The symbol $\operatorname{card}(A)$ stands for the cardinality of the set $A$. Since the domain of all considered functions is the unit interval, then the notation connected with intervals $[a, b]$ $((a, b)$, etc. $)$ will in fact mean intersection of this interval and $[0,1]$

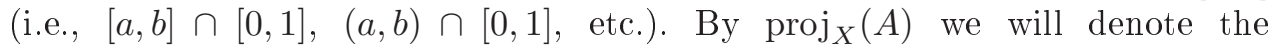
$x$-projection of $A$.

If $\mathcal{F}$ is a family of sets and $Z$ is a set, then $\mathcal{F} \subset Z$ means that $A \subset Z$ for each $A \in \mathcal{F}$.

If $A \subset[0,1]$, then the density of a set $A$ at $x_{0}$ (with respect to the Lebesgue measure) will be denoted by $d\left(A, x_{0}\right)$.

Symbols $\operatorname{Fix}(f)$ and $\mathrm{C}(f)$ will stand for the sets of all fixed points of function $f$ and of all continuity points of $f$, respectively. Moreover, if $A \subset[0,1]$, then we will write $f\lceil A$ for the restriction of $f$ to the set $A$. The notation $A \underset{f}{\rightarrow} B$ (a set $A f$-covers $B$ ) will mean that $B \subset f(A)$.

Following [11, we adopt the notions related to $f$-bundles. A pair $(\mathfrak{F}, \mathcal{J})=B_{f}$, where $\mathfrak{F}$ is a family of pairwise disjoint (nonsingletons) continuums in $[0,1]$ and $\mathcal{J} \subset[0,1]$ is a connected set such that $A \rightarrow \mathcal{f}$ for any $A \in \mathfrak{F}$, is called an $f$-bundle. Moreover, if we additionally assume that $A \subset \mathcal{J}$ for all $A \in \mathfrak{F}$, 
then such an $f$-bundle is called an $f$-bundle with dominating fibre. For brevity, we adopt the convention that $\operatorname{card}\left(B_{f}\right)=\operatorname{card}(\mathfrak{F})$.

Let $\varepsilon>0$ and $n \in \mathbb{N}$. A set $M \subset \bigcup \mathfrak{F}$ is $\left(B_{f}, n, \varepsilon\right)$-separated if for each $x, y \in M$, $x \neq y$ there is $0 \leqslant i<n$ such that $f^{i}(x), f^{i}(y) \in \mathcal{J}$ and $\rho\left(f^{i}(x), f^{i}(y)\right)>\varepsilon$.

The entropy of the $f$-bundle $B_{f}$ is defined in the following way

where

$$
h\left(B_{f}\right)=\lim _{\varepsilon \rightarrow 0} \limsup _{n \rightarrow \infty}\left[\frac{1}{n} \log \left(s_{n}^{B_{f}}(\varepsilon)\right)\right],
$$

$$
s_{n}^{B_{f}}(\varepsilon)=\max \left\{\operatorname{card}(M): M \subset X \text { is }\left(B_{f}, n, \varepsilon\right) \text {-separated set }\right\} .
$$

A sequence of $f$-bundles $B_{f}^{k}=\left(\mathfrak{F}_{k}, \mathcal{J}_{k}\right)$ converges to a point $x_{0}\left(B_{f}^{k} \underset{k \rightarrow \infty}{\longrightarrow} x_{0}\right)$, if for any $\varepsilon>0$ there exists $k_{0} \in \mathbb{N}$ such that

$\bigcup \mathfrak{F}_{k} \subset\left(x_{0}-\varepsilon, x_{0}+\varepsilon\right)$ and $\left(f\left(x_{0}\right)-\varepsilon, f\left(x_{0}+\varepsilon\right)\right) \cap \mathcal{J}_{k} \neq \emptyset \quad$ for any $k \geqslant k_{0}$. Let

$$
\mathrm{E}_{f}\left(x_{0}\right)=\left\{\limsup _{n \rightarrow \infty} h\left(B_{f}^{n}\right): B_{f}^{n} \underset{n \rightarrow \infty}{\longrightarrow} x_{0}\right\} .
$$

The notions of a pseudo-fixed point and an $\infty$-entropy point have been introduced in [3]. We say that a point $x_{0}$ is a pseudo-fixed point of the function $f$ (denoted by $x_{0} \in \mathrm{pFix}(f)$ ), if every neighbourhood of $x_{0}$ contains a fixed point of $f$.

We will say that a point $x_{0}$ is an $\infty$-entropy point of $f$ if $\infty \in \mathrm{E}_{\mathrm{f}}\left(\mathrm{x}_{0}\right)$ and $x_{0} \in$ $\operatorname{pFix}(f)$. Obviously, we have that each $\infty$-entropy point of $f$ is also an entropy point of $f$, in the sense of definition introduced in [11]. The set of all $\infty$-entropy points of $f$ will be denoted by $\mathrm{E}_{\infty}(f)$. Simultaneously, we will denote by $\mathrm{E}_{0}(f)$ the set of all points $x$ such that $\mathrm{E}_{f}(x)=\{0\}$. For our considerations, it will also be convenient to adopt the denotation $\mathrm{E}_{\infty}^{A}=\left\{g \in \mathcal{A}: a \in \mathrm{E}_{\infty}(g)\right.$ for each $\left.a \in A\right\}$. In other words, $\mathrm{E}_{\infty}^{A}$ is the family of all (almost continuous) functions $g$ such that each point $a \in A$ is an $\infty$-entropy point of $g$.

\section{Definitions and basic properties}

The main idea of the concept of a new notion extremely chaotic point of some function has been presented in the first part of the paper. Thus, go directly to the definition.

DEFinition 2.1. A point $x_{0}$ is said to be an extremely chaotic point of $f$ if there exist sets $A, B \subset[0,1]$ such that $\mathrm{d}\left(\bar{A}, x_{0}\right)=\frac{1}{2}=\mathrm{d}\left(\bar{B}, x_{0}\right)$ and the following conditions are fulfilled:

(I) $A \subset \mathrm{E}_{0}(f)$ and $f \uparrow \bar{A}$ is continuous at $x_{0}$;

(II) there exists $\sigma>0$ such that $B_{u}(f, \sigma) \subset E_{\infty}^{B \cup\left\{x_{0}\right\}}$. 
It is easy to see (accepting denotations used in the above definition) that if $x_{0}$ is an extremly chaotic point, then $x_{0} \in \bar{A} \cap \bar{B}$.

The next proposition is useful for proofs of some facts as well as for explanation of the behaviour of function on the closure of sets consisting of $\infty$-entropy points. Before proving the announced proposition, we recall the theorem (formulated as a lemma), which will also be useful in further considerations.

LEMMA 2.2 ([11]). Let $f$ be an arbitrary function and $B_{f}$ be an $f$-bundle with dominating fibre. Then, $h\left(B_{f}\right) \geq \log \left(\operatorname{card}\left(B_{f}\right)\right)$ whenever $B_{f}$ is finite, and $h\left(B_{f}\right)=+\infty$ whenever $B_{f}$ is infinite.

Proposition 2.3. If $f\left(x_{0}\right) \in \mathrm{L}_{B}\left(f, x_{0}\right)$ and $B \subset \mathrm{E}_{\infty}(f)$, then $x_{0} \in \mathrm{E}_{\infty}(f)$.

P r o o f. Let us first notice that from the assumption $f\left(x_{0}\right) \in \mathrm{L}_{B}\left(f, x_{0}\right)$ it follows that $x_{0} \in \bar{B}$. So, let $\left\{x_{n}\right\} \subset B$ be a sequence such that

$$
x_{n} \in\left(x_{0}-\frac{1}{n}, \quad x_{0}+\frac{1}{n}\right), \quad x_{n} \longrightarrow x_{0} \quad \text { and } \quad f\left(x_{n}\right) \longrightarrow f\left(x_{0}\right) .
$$

Since $x_{n} \in \operatorname{pFix}(f)$, we infer that $x_{0} \in \operatorname{pFix}(f)$. So, it is sufficient to show that

$$
\infty \in \mathrm{E}_{f}\left(x_{0}\right) .
$$

Taking Lemma 2.2 into account, in order to prove (2), we shall show that for each positive integer $n$, there exists $f$-bundle

$$
B_{f}^{n}=\left(\mathfrak{F}_{n}, \mathcal{J}_{n}\right)
$$

with dominating fibre such that

$$
\begin{array}{r}
\mathfrak{F}_{n} \subset\left(x_{0}-\frac{1}{n}, x_{0}+\frac{1}{n}\right),\left(f\left(x_{0}\right)-\frac{1}{n}, f\left(x_{0}\right)+\frac{1}{n}\right) \cap \mathcal{J}_{n} \neq \emptyset \\
\text { and } \operatorname{card}\left(B_{f}^{n}\right)>2^{n} .
\end{array}
$$

Obviously, $x_{n} \in \mathrm{E}_{\infty}(f)$, for each positive integer $n$. The definition of the $\infty$-entropy point and (11) permit to conclude that for each $n \in \mathbb{N}$ there exists $f$-bundle

$$
\mathrm{B}_{f}\left(x_{n}\right)=\left(\mathfrak{F}_{n}, \mathcal{J}_{n}\right)
$$

such that

$$
\begin{array}{r}
\mathfrak{F}_{n} \subset\left(x_{0}-\frac{1}{n}, x_{0}+\frac{1}{n}\right),\left(f\left(x_{0}\right)-\frac{1}{n}, f\left(x_{0}\right)+\frac{1}{n}\right) \cap \mathcal{J}_{n} \neq \emptyset \\
\text { and } \operatorname{card}\left(B_{f}^{n}\right)>2^{n} .
\end{array}
$$

To prove (3), it is sufficient to put

$$
B_{f}^{n}=B_{f}\left(x_{n}\right) .
$$

Before going to main results, it is worth noting the following proposition which obviously directs our considerations to discontinuous functions. 
Proposition 2.4. If $x_{0}$ is an extremely chaotic point of $f$, then $x_{0} \notin C(f)$.

P r o of. In order to prove this proposition, we only need to use condition (II).

Conversely, suppose that

$$
x_{0} \in C(f) .
$$

So, let us fix $\sigma>0$. According to the continuity of $f$ at $x_{0}$, there exists $\delta>0$ such that

$$
f\left(\left[x_{0}-\delta, x_{0}+\delta\right]\right) \subset\left(f\left(x_{0}\right)-\frac{\sigma}{2}, f\left(x_{0}\right)+\frac{\sigma}{2}\right) .
$$

Now, we shall define a function $\varphi:[0,1] \rightarrow[0,1]$ in the following way:

$$
\begin{aligned}
& \varphi(x)=f\left(x_{0}\right), \text { for } x \in\left[x_{0}-\frac{\delta}{2}, x_{0}+\frac{\delta}{2}\right] ; \\
& \varphi(x)=f(x), \text { for } x \notin\left[x_{0}-\delta, x_{0}+\delta\right] ;
\end{aligned}
$$

$\varphi$ is linear in each interval $\left[x_{0}-\delta, x_{0}+\delta\right] \backslash\left[x_{0}-\frac{\delta}{2}, x_{0}+\frac{\delta}{2}\right]$.

(Let us note that the above definition of $\varphi$ includes all the cases $x_{0} \in(0,1), x_{0}=0$ or $\left.x_{0}=1.\right)$

So, $\varphi \in \mathcal{A}$ ([6], [7]) and $\varphi \in B_{u}(f, \sigma)$. Obviously, $x_{0} \notin E_{\infty}(\varphi)$.

An immediate consequence of Proposition 2.4 is the statement that no continuous function has an extremely chaotic point. On the other hand, there exist examples of discontinuous (but almost continuous) functions with no extremely chaotic point. It is sufficient to consider a function $f:[0,1] \rightarrow[0,1]$ defined in the following way:

$$
f(0)=\frac{1}{2}, \quad f(x)=\frac{1}{4} \sin \frac{1}{x}+\frac{3}{4} \text { for } x \in\left(0, \frac{2}{5 \pi}\right], f(x)=1 \text { for } x \in\left[\frac{2}{5 \pi}, 1\right] .
$$

Then

$$
h\left(f^{2}\right)=0 .
$$

In order to avoid misunderstandings and to make this paper more readable, we present some lemmas proved as theorems in other papers.

LEMMa 2.5 ([13]). If nondegenerate Hausdorff space $X$ has a fixed point property, then each almost continuous function $g: X \rightarrow X$ has a fixed point.

Lemma 2.6 ([1]). If $a, b \in[0,1]$ and $f:(a, b) \rightarrow \mathbb{R}$ is almost continuous,

$$
y \in L^{+}(f, a), \quad z \in L^{-}(f, b),
$$

then the functions

$$
f_{1}:[a, b) \rightarrow \mathbb{R}, \quad f_{2}:(a, b] \rightarrow \mathbb{R}, \quad f_{3}:[a, b] \rightarrow \mathbb{R}
$$

such that

$$
\begin{gathered}
f_{1}(x)=f_{2}(x)=f_{3}(x)=f(x) \text { for } x \in(a, b), \\
f_{1}(a)=f_{3}(a)=y \quad \text { and } \quad f_{2}(b)=f_{3}(b)=z
\end{gathered}
$$

are almost continuous. 


\section{Main results}

Following the results contained in [3, it seems to be interesting to consider a family of functions having common chaotic points. This leads us to following definitions.

Definition 3.1. We say that a nonempty family of functions $\mathcal{F}$ has the extremely chaotic property at $x_{0}$ if $x_{0}$ is an extremely chaotic point of each function belonging to $\mathcal{F}$. We say that a nonempty family of functions $\mathcal{F}$ has the extremely chaotic point property if there exists a point $x_{0}$ such that $\mathcal{F}$ has the extremely chaotic property at $x_{0}$.

On account of the example presented after Proposition 2.4, the following question, "Is it possible to modify any almost continuous function to a function having extremely chaotic point?" seems to be interesting. What is more, one can ask how many modified functions one can obtain in such a way that they create a family of functions having the extremely chaotic point property. Certainly, first, we have to explain what is by modifying meant.

DEFinition 3.2. We say that a function $\varphi^{\star}$ is an $\varepsilon$-modification of $\varphi(\varepsilon>0)$ or that $\varphi$ is $\varepsilon$-modifiable to a function $\varphi^{\star}$ if $\operatorname{dia}\left\{x \in[0,1]: \varphi(x) \neq \varphi^{\star}(x)\right\}<\varepsilon$.

THEOREM 3.3. For each function $f \in \mathcal{A}$ and each $\mathcal{T}_{\Gamma}$-neighbourhood $U$ of $f$ and $\varepsilon>0$, there exists an uncountabl@ family $\mathcal{F} \subset \mathcal{A}$ consisting of functions being $\varepsilon$-modifications of $f$ such that $\mathcal{F}$ has extremely chaotic point property and $\mathcal{F} \subset U$.

The initial part of the proof. The starting point of this proof as well as the proof of the next theorem will be a construction of some almost continuous function $\psi^{\mu, \nu}$, where $\mu, \nu \in[0,1]$.

Let $\mathcal{M}=\left\{M_{\alpha}\right\}_{\alpha<\mathfrak{c}}$ be a transfinite sequence of all closed sets being a subset of $[0,1]^{2}$ and such that

$$
\operatorname{Int}\left(\operatorname{proj}_{X}\left(M_{\alpha}\right)\right) \neq \emptyset
$$

(and consequently, $\left.\operatorname{card}\left(\operatorname{proj}_{X}\left(M_{\alpha}\right)\right)=\mathfrak{c}\right)$, for each $\alpha<\mathfrak{c}$. Put $H=\{0,1\}$ and let $\psi^{\mu, \nu}(0)=\mu$ and $\psi^{\mu, \nu}(1)=\nu$ for $\mu, \nu \in[0,1]$.

Fix the numbers $\mu, \nu \in[0,1]$. Now, we define a function $\psi^{\mu, \nu}$ on some subset of $[0,1]$ inductively.

Let $x_{0} \in \operatorname{proj}_{X}\left(M_{0}\right) \backslash H$. Fix $y_{0} \in[0,1]$ such that $\left(x_{0}, y_{0}\right) \in M_{0}$ and put $\psi^{\mu, \nu}\left(x_{0}\right)=y_{0}$.

\footnotetext{
${ }^{1}$ In our proof we will show that there exists a suitable family of the power continuum. Slight changes in the construction of these functions permit to obtain a family of the power greater than continuum. Since it has no influence on the essence of the considerations, we have introduced such a form of the theorem and of the proof in order to facilitate the reading.
} 
Let us suppose that we have defined $\left(x_{\beta}, \psi^{\mu, \nu}\left(x_{\beta}\right)\right) \in M_{\beta}$ for $\beta<\alpha<\mathfrak{c}$ such that

$$
x_{\beta} \in \operatorname{proj}_{X}\left(M_{\beta}\right) \backslash\left(H \cup\left\{x_{\gamma}, \psi^{\mu, \nu}\left(x_{\gamma}\right): \gamma<\beta\right\}\right) .
$$

Obviously, the sets $A_{\alpha}:=\left\{x_{\beta}: \beta<\alpha\right\}$ and $B_{\alpha}:=\left\{\psi^{\mu, \nu}\left(x_{\beta}\right): \beta<\alpha\right\}$ are of size $<\mathfrak{c}$. So, let

$$
x_{\alpha} \in \operatorname{proj}_{X}\left(M_{\alpha}\right) \backslash\left(H \cup A_{\alpha} \cup B_{\alpha}\right) .
$$

Then, there is $y_{\alpha} \in[0,1]$ such that $\left(x_{\alpha}, y_{\alpha}\right) \in M_{\alpha}$. Put $\psi^{\mu, \nu}\left(x_{\alpha}\right)=y_{\alpha}$.

Let $\psi^{\mu, \nu}(x)=0$, for all points $x \in[0,1] \backslash\left(H \cup\left\{x_{\alpha}: \alpha<\mathfrak{c}\right\}\right)$.

We will show that

$$
\psi^{\mu, \nu}(\mathfrak{I})=[0,1], \text { for each nondegenerated interval } \mathfrak{I} \subset[0,1] .
$$

Fix $d \in[0,1]$. There exist $\gamma_{0}<\mathfrak{c}$ and a closed interval $\mathfrak{I}_{1} \subset \mathfrak{I}$ such that

$$
\mathfrak{I}_{1} \times\{d\} \in M_{\gamma_{0}} \in \mathcal{M} \text {. }
$$

According to the definition of $\psi^{\mu, \nu}$, we have $x_{\gamma_{0}} \in \mathfrak{I}$ and $\psi^{\mu, \nu}\left(x_{\gamma_{0}}\right)=d$. The proof of (4) is finished.

What is left is to show that

$$
\psi^{\mu, \nu} \in \mathcal{A} \text {. }
$$

Notice that the family of all blocking sets is contained in $\mathcal{M}$ ([6], 7]), which proves (5) 3

The main part of the proof. Let $\varepsilon>0$ and let $U$ be $\mathcal{T}_{\Gamma}$-neighbourhood of $f$. According to Lemma 2.5 there exists $x_{0} \in \operatorname{Fix}(f)$. There is no loss of generality in assuming that $x_{0} \in(0,1)$ (in the case if either $x_{0}=0$ or $x_{0}=1$, the proof runs analogously). Fix $\varepsilon_{0} \in(0, \varepsilon)$ such that $\left[x_{0}-\varepsilon_{0}, x_{0}+\varepsilon_{0}\right] \subset(0,1)$ and $\left[x_{0}-\varepsilon_{0}, x_{0}+\varepsilon_{0}\right]^{2} \subset U$.

Since $f$ is a Darboux function ([7], [13]), there exist $a_{0} \in\left(x_{0}-\frac{\varepsilon_{0}}{4}, x_{0}\right)$, $b_{0} \in\left(x_{0}, x_{0}+\frac{\varepsilon_{0}}{4}\right)$ such that $f\left(a_{0}\right), f\left(b_{0}\right) \in\left(x_{0}-\varepsilon_{0}, x_{0}+\varepsilon_{0}\right)$.

Let us consider sequences of closed intervals

such that:

$$
\left\{\left[a_{n}^{1}, b_{n}^{1}\right]\right\}_{n=1}^{\infty}, \quad\left\{\left[a_{n}^{2}, b_{n}^{2}\right]\right\}_{n=1}^{\infty}
$$

$$
\begin{aligned}
& a_{0}<a_{1}^{1}<b_{1}^{1}<\cdots<a_{n}^{1}<b_{n}^{1}<\cdots<x_{0}(n=1,2, \ldots) ; \\
& b_{0}>b_{1}^{2}>a_{1}^{2}>\cdots>b_{n}^{2}>a_{n}^{2}>\cdots>x_{0}(n=1,2, \ldots) ; \\
& \mathrm{d}\left(\bigcup_{n=1}^{\infty}\left(\left[a_{n}^{1}, b_{n}^{1}\right] \cup\left[a_{n}^{2}, b_{n}^{2}\right]\right), x_{0}\right)=\frac{1}{2}=\mathrm{d}\left(\bigcup_{n=1}^{\infty}\left(\left[b_{n}^{1}, a_{n+1}^{1}\right] \cup\left[b_{n+1}^{2}, a_{n}^{2}\right]\right), x_{0}\right) . \\
& \quad \operatorname{Put} \\
& A=\bigcup_{n=1}^{\infty}\left(\left[a_{n}^{1}, b_{n}^{1}\right] \cup\left[a_{n}^{2}, b_{n}^{2}\right]\right) .
\end{aligned}
$$

\footnotetext{
${ }^{2}$ From now on, if we write $\psi^{\mu, \nu}(\mu, \nu \in[0,1])$, then we mean the function constructed above.
} 
Now, we will define the function $\varphi_{t}:[0,1] \rightarrow[0,1]$, for $t \in\left[x_{0}-\frac{\varepsilon_{0}}{4}, x_{0}+\frac{\varepsilon_{0}}{4}\right]$. Let

$$
\begin{aligned}
& \varphi_{t}(x)=t, \quad \text { for } x \in A \cup\left\{x_{0}\right\} ; \\
& \varphi_{t}(x)=f(x), \text { for } x \notin\left(a_{0}, b_{0}\right) ; \\
& \varphi_{t}(x)=\max \left(x_{0}-\varepsilon_{0}, \min \left(x_{0}+\varepsilon_{0}, \psi^{t, t}\left(\frac{x-b_{n}^{1}}{a_{n+1}^{1}-b_{n}^{1}}\right)\right)\right), \\
& \quad \text { for } x \in\left[b_{n}^{1}, a_{n+1}^{1}\right],(n=1,2, \ldots) ; \\
& \varphi_{t}(x)=\max \left(x_{0}-\varepsilon_{0}, \min \left(x_{0}+\varepsilon_{0}, \psi^{t, t}\left(\frac{x-b_{n+1}^{2}}{a_{n}^{2}-b_{n+1}^{2}}\right)\right)\right), \\
& \quad \text { for } x \in\left[b_{n+1}^{2}, a_{n}^{2}\right],(n=1,2, \ldots) ;
\end{aligned}
$$

$\varphi_{t}$ is linear in each interval $\left[a_{0}, a_{1}^{1}\right],\left[b_{1}^{2}, b_{0}\right]$.

Note that

$$
\begin{aligned}
\left\{x \in[0,1]: f(x) \neq \varphi_{t}(x)\right\} \subset\left(a_{0}, b_{0}\right) \subset & \left(x_{0}-\frac{\varepsilon_{0}}{4}, x_{0}+\frac{\varepsilon_{0}}{4}\right), \\
& \text { for } t \in\left[x_{0}-\frac{\varepsilon_{0}}{4}, x_{0}+\frac{\varepsilon_{0}}{4}\right],
\end{aligned}
$$

which means that $\varphi_{t}$ is $\varepsilon$-modification of $f$ (actually, it is $\varepsilon_{0}$-modification of $f$ ).

Put $\mathcal{F}_{f}=\left\{\varphi_{t}: t \in\left[x_{0}-\frac{\varepsilon_{0}}{4}, x_{0}+\frac{\varepsilon_{0}}{4}\right]\right\}$. Certainly, the power of $\mathcal{F}_{f}$ is continuum. Now, we shall show that

$$
\mathcal{F}_{f} \subset \mathcal{A} \text {. }
$$

Fix $\varphi_{t_{0}} \in \mathcal{F}_{f}$ (i.e., $\left.t_{0} \in\left[x_{0}-\frac{\varepsilon_{0}}{4}, x_{0}+\frac{\varepsilon_{0}}{4}\right]\right)$. Now, let us remark ([6], [7]) that the following functions are almost continuous:

$$
\begin{aligned}
& \varphi_{t_{0}} \uparrow\left[0, a_{0}\right], \varphi_{t_{0}} \uparrow\left[a_{0}, a_{1}^{1}\right], \quad \varphi_{t_{0}} \uparrow\left[b_{1}^{2}, b_{0}\right], \varphi_{t_{0}} \uparrow\left[b_{0}, 1\right] ; \\
& \varphi_{t_{0}} \uparrow\left[a_{n}^{1}, b_{n}^{1}\right], \varphi_{t_{0}} \uparrow\left[b_{n}^{1}, a_{n+1}^{1}\right], \varphi_{t_{0}} \uparrow\left[a_{n}^{2}, b_{n}^{2}\right], \varphi_{t_{0}} \uparrow\left[a_{n+1}^{2}, b_{n}^{2}\right],(n=1,2, \ldots) .
\end{aligned}
$$

Then, ([6], [7]) the functions $\varphi_{t} \uparrow\left[0, x_{0}\right), \varphi_{t} \uparrow\left(x_{0}, 1\right]$ are almost continuous functions and, according to Lemma 2.6] $\varphi_{t} \uparrow\left[0, x_{0}\right], \varphi_{t} \uparrow\left[x_{0}, 1\right]$ are almost continuous functions. Consequently, the proof of (6) is finished.

It is easy to see that

Now, let us put

$$
\mathcal{F}_{f} \subset U .
$$

$$
\begin{aligned}
B_{t}=\left\{x \in\left[a_{1}^{1}, b_{1}^{2}\right] \backslash\left(A \cup\left\{x_{0}\right\}\right): \varphi_{t}(x) \in\right. & \left.\left(x_{0}-\frac{\varepsilon_{0}}{20}, x_{0}+\frac{\varepsilon_{0}}{20}\right)\right\}, \\
& \text { for } t \in\left[x_{0}-\frac{\varepsilon_{0}}{4}, x_{0}+\frac{\varepsilon_{0}}{4}\right] .
\end{aligned}
$$

We shall show that

$$
\overline{B_{t}}=\left\{x_{0}\right\} \cup \bigcup_{n=1}^{\infty}\left(\left[b_{n}^{1}, a_{n+1}^{1}\right] \cup\left[b_{n+1}^{2}, a_{n}^{2}\right]\right), \quad \text { for } \quad t \in\left[x_{0}-\frac{\varepsilon}{4}, x_{0}+\frac{\varepsilon}{4}\right] \text {. }
$$


For this purpose, it is sufficient to show that $(x-\delta, x+\delta) \cap B_{t} \neq \emptyset$, for each

$$
x \in \bigcup_{n=1}^{\infty}\left(\left[b_{n}^{1}, a_{n+1}^{1}\right] \cup\left[b_{n+1}^{2}, a_{n}^{2}\right]\right), \delta>0 \quad \text { and } \quad t \in\left[x_{0}-\frac{\varepsilon_{0}}{4}, x_{0}+\frac{\varepsilon_{0}}{4}\right] .
$$

So, let us fix

$$
x \in \bigcup_{n=1}^{\infty}\left(\left[b_{n}^{1}, a_{n+1}^{1}\right] \cup\left[b_{n+1}^{2}, a_{n}^{2}\right]\right), \delta>0 \quad \text { and } \quad t_{1} \in\left[x_{0}-\frac{\varepsilon_{0}}{4}, x_{0}+\frac{\varepsilon_{0}}{4}\right] .
$$

In order to simplify the considerations and without loss of generality, one can assume that $x \in\left(b_{n_{x}}^{1}, a_{n_{x}+1}^{1}\right)$, for some $n_{x} \in \mathbb{N}$ and, moreover,

$$
(x-\delta, x+\delta) \subset\left(b_{n_{x}}^{1}, a_{n_{x}+1}^{1}\right) .
$$

The equality (4) and definition of $\varphi_{t_{1}}$ permit to infer that there is

$$
y \in(x-\delta, \quad x+\delta)
$$

such that

$$
\varphi_{t_{1}}(y) \in\left(x_{0}-\frac{\varepsilon_{0}}{20}, x_{0}+\frac{\varepsilon_{0}}{20}\right),
$$

which gives $y \in B_{t_{1}}$. The proof of (8) (and at the same time the proof of (7)) is finished.

Our assumptions connected with sequences $\left\{\left[b_{n}^{1}, a_{n+1}^{1}\right]\right\}_{n=1}^{\infty}\left\{\left[b_{n+1}^{2}, a_{n}^{2}\right]\right\}_{n=1}^{\infty}$ and (7) give

$$
\mathrm{d}\left(\overline{B_{t}}, x_{0}\right)=\frac{1}{2} .
$$

Certainly, the set $\operatorname{Int}(A)$ fulfils the condition (I) with respect to

$$
\varphi_{t}\left(t \in\left[x_{0}-\frac{\varepsilon_{0}}{4}, x_{0}+\frac{\varepsilon_{0}}{4}\right]\right) \text { and } x_{0} .
$$

What is left is to show that

the condition (II) of Definition 2.1 is fulfiled for each function $\varphi_{t}$ with respect to $B_{t}$, for any $t \in\left[x_{0}-\frac{\varepsilon_{0}}{4}, x_{0}+\frac{\varepsilon_{0}}{4}\right]$.

Fix $t_{*} \in\left[x_{0}-\frac{\varepsilon_{0}}{4}, x_{0}+\frac{\varepsilon_{0}}{4}\right]$ and let $\sigma=\frac{\varepsilon_{0}}{5}, \eta \in B_{u}\left(\varphi_{t_{*}}, \sigma\right)$. We will first show that

$$
z \text { is an } \infty \text {-entropy point of } \eta \text {, for each } z \in B_{t_{*}} \text {. }
$$

So, let $z_{0} \in B_{t_{*}}$ and suppose that $z_{0} \in\left[b_{m}^{1}, a_{m+1}^{1}\right]$ for some positive integer $m$ (if $z_{0} \in\left[b_{m+1}^{2}, a_{m}^{2}\right]$, for some $m \in \mathbb{N}$, the proof runs analogously). There is no loss of generality in assuming that $z_{0} \neq a_{m+1}^{1}$. 


\section{ON POINTS OF EXTREME CHAOS FOR ALMOST CONTINUOUS FUNCTIONS}

Then, there exist sequences $\left\{s_{n}\right\}_{n=1}^{\infty},\left\{z_{n}\right\}_{n=1}^{\infty} \subset\left(z_{0}, a_{m+1}^{1}\right)$ such that

$$
\begin{aligned}
& a_{m+1}^{1}>z_{1}>s_{1}>z_{2}>s_{2}>\cdots ; \\
& z_{n} \searrow z_{0} ; \\
& \varphi_{t_{*}}\left(s_{i}\right)=x_{0}-\varepsilon_{0}, \\
& \varphi_{t_{*}}\left(z_{i}\right)=x_{0}+\varepsilon_{0} \quad \text { for } \quad i \in \mathbb{N} .
\end{aligned}
$$

Since $\eta \in B_{u}\left(\varphi_{t_{*}}, \sigma\right)$, we have

$$
\eta\left(z_{0}\right) \in\left(x_{0}-\frac{\varepsilon_{0}}{4}, x_{0}+\frac{\varepsilon_{0}}{4}\right) .
$$

Moreover, it is easy to see that

$$
\eta\left(s_{i}\right) \in\left[0, x_{0}-\frac{4 \varepsilon_{0}}{5}\right] \quad \text { and } \quad \eta\left(z_{i}\right) \in\left[x_{0}+\frac{4 \varepsilon_{0}}{5}, 1\right] \quad \text { for } \quad i \in \mathbb{N} .
$$

Next, one can observe that

$$
\left[s_{i}, z_{i}\right] \subset\left(a_{0}, b_{0}\right) \subset\left(x_{0}-\frac{\varepsilon_{0}}{4}, x_{0}+\frac{\varepsilon_{0}}{4}\right), \quad \text { for } \quad i \in \mathbb{N} .
$$

Since $\eta \in \mathcal{A}$, from (11), it follows that

$$
\left[s_{i}, z_{i}\right] \underset{\eta}{\rightarrow}\left[s_{j}, z_{j}\right], \quad \text { for any } i, j \in \mathbb{N} \text {. }
$$

Now, taking (13) and the fact that $\eta$ is a connectivity function into account, we may infer

$$
\operatorname{Fix}(\eta) \cap\left[s_{i}, z_{i}\right] \neq \emptyset, \quad \text { for any } \quad i \in \mathbb{N} .
$$

On account of the above remark, we have

$$
z_{0} \in \operatorname{pFix}(\eta)
$$

The next step of our proof will be connected with the definition of suitable $\eta$-bundles. Put $\mathcal{J}_{k}=\left[x_{0}-\frac{\varepsilon_{0}}{4}, x_{0}+\frac{\varepsilon_{0}}{4}\right](k=1,2, \ldots)$ and let

$$
\begin{aligned}
K_{1} & =\left\{\left[s_{1}, z_{1}\right],\left[s_{2}, z_{2}\right]\right\} ; \\
K_{2} & =\left\{\left[s_{3}, z_{3}\right], \ldots,\left[s_{6}, z_{6}\right]\right\} ; \\
K_{3} & =\left\{\left[s_{7}, z_{7}\right], \ldots,\left[s_{14}, z_{14}\right]\right\}, \ldots
\end{aligned}
$$

Generally, $K_{k}$ denotes the family consisting of consecutive $2^{k}$ intervals which were not used in previous families of intervals, starting with $K_{1}=\left\{\left[s_{1}, z_{1}\right],\left[s_{2}, z_{2}\right]\right\}$.

Set $\mathfrak{B}_{\eta}^{k}=\left(K_{k}, \mathcal{J}_{k}\right)$, for $k=1,2, \ldots$ From (12) and (13), it may be concluded that $\mathfrak{B}_{\eta}^{k}$ is an $\eta$-bundle with the dominating fibre $(k=1,2, \ldots)$. According to Lemma 2.2 we have

$$
\mathrm{h}\left(\mathfrak{B}_{\eta}^{k}\right) \geq k
$$


Using definition of $\mathfrak{B}_{\eta}^{k}$ and (10), it may be concluded that $\mathfrak{B}_{\eta}^{k}$ convengers to a point $z_{0}$.

Consequently, $\infty \in \mathrm{E}_{\eta}\left(z_{0}\right)$ which, according to (14), finishes the proof of the fact that $z_{0}$ is an $\infty$-entropy point of $\eta$.

The method of the proof of (9) works for the proof of the relationship $x_{0}$ is an $\infty$-entropy point of $\eta$.

The OREM 3.4. In the bi-topological space $\left(\mathcal{A}, \mathcal{T}_{\Gamma}, \mathcal{T}_{u}\right)$, every family of functions having extremely chaotic property at $x_{0}$ is $\left(\mathcal{T}_{\Gamma}, \mathcal{T}_{u}\right)$ - nowhere dense set.

P r o of. In many parts of this proof, the ideas of considerations are similar to those in the proof of Theorem 3.3. In order to avoid an artificial lengthening of the proof, we restrict the suitable considerations only to a signaling the essential facts.

Let $\mathfrak{F} \subset \mathcal{A}$ be a nonempty family having extremely chaotic property at $x_{0}$.

Let $W \in \mathcal{T}_{\Gamma} \backslash\{\emptyset\}$ and let us fix $f \in W$. There is no loss of generality in assuming that $x_{0} \in(0,1)$ (in the case if either $x_{0}=0$ or $x_{0}=1$, it is sufficient to restrict the proof to the one-side considerations). Certainly, $W$ contains some continuous function $\varphi$ defined on $[0,1]$. So, let $\varepsilon>0$ be a number such that

$$
\left[x_{0}-\varepsilon, x_{0}+\varepsilon\right] \times\left[\varphi\left(x_{0}\right)-\varepsilon, \varphi\left(x_{0}\right)+\varepsilon\right] \subset W .
$$

Obviously, we have two possibilities:

$$
\begin{aligned}
& 1^{\mathrm{o}} \varphi\left(x_{0}\right)=x_{0}, \text { then we put } \sigma=\varepsilon . \\
& \left.2^{\mathrm{o}} \varphi\left(x_{0}\right) \neq x_{0} \text { (for simplicity's sake, we will assume that } \varphi\left(x_{0}\right)>x_{0}\right) .
\end{aligned}
$$

In this case, let $\sigma \in(0, \varepsilon)$ be a number such that

$$
\left[x_{0}-\sigma, x_{0}+\sigma\right] \times\left[\varphi\left(x_{0}\right)-\sigma, \varphi\left(x_{0}\right)+\sigma\right] \cap\{(x, y): x=y\}=\emptyset .
$$

In both cases, let $\alpha \in\left(x_{0}-\frac{\sigma}{2}, x_{0}\right), \beta \in\left(x_{0}, x_{0}+\frac{\sigma}{2}\right)$ be numbers such that

$$
\varphi([\alpha, \beta]) \subset\left(\varphi\left(x_{0}\right)-\frac{\sigma}{2}, \varphi\left(x_{0}\right)+\frac{\sigma}{2}\right) .
$$

Now, we define a function $\xi:[0,1] \rightarrow[0,1]$ in the following way:

$$
\begin{aligned}
& \xi(x)=\varphi(x), \text { for } x \in[0, \alpha] \cup[\beta, 1] ; \\
& \xi(x)=\max \left(\varphi\left(x_{0}\right)-\frac{\sigma}{2}, \min \left(\varphi\left(x_{0}\right)+\frac{\sigma}{2}, \psi^{\varphi(\alpha), \varphi(\beta)}\left(\frac{x-\alpha}{\beta-\alpha}\right)\right)\right) \text { for } x \in[\alpha, \beta],
\end{aligned}
$$

where $\psi^{\varphi(\alpha), \varphi(\beta)}$ is a function defined in the Initial part of the proof of Theorem 3.3 . 
Let us remark that $\xi(\alpha)=\varphi(\alpha), \xi(\beta)=\varphi(\beta)$ and for each nondegenerated interval $\mathfrak{I} \subset[\alpha, \beta]$ the equality $\xi(\mathfrak{I})=\left[\varphi\left(x_{0}\right)-\frac{\sigma}{2}, \varphi\left(x_{0}\right)+\frac{\sigma}{2}\right]$ takes place. Similarly as in the proof of Theorem 3.3 one can prove that $\xi \in \mathcal{A}$ and $\xi \in W$. Put

$\sigma_{0}:=\min \left(\frac{\sigma}{4}, \inf \left\{\varrho\left((x, \varphi(x)),[0,1]^{2} \backslash W\right): x \in[0,1]\right\}\right)>0$ if $[0,1]^{2} \backslash W \neq \emptyset$

and

$\sigma_{0}:=\frac{\sigma}{4}$, if $[0,1]^{2} \backslash W=\emptyset$.

Set $V=\mathrm{B}_{u}\left(\xi, \sigma_{0}\right)$. First, we will show that

$$
V \subset W \text {. }
$$

It is sufficient to prove this assertion, under the assumption $W \neq[0,1]^{2}$.

Put $\tau_{\varphi}:=\inf \left\{\varrho\left((x, \varphi(x)),[0,1]^{2} \backslash W\right): x \in[0,1]\right\}$. First, we will prove

$$
\varrho\left(\left(u_{1}, u_{2}\right), \xi\right) \geq \sigma_{0}, \quad \text { for each } \quad\left(u_{1}, u_{2}\right) \in[0,1]^{2} \backslash W=F .
$$

So, let us fix $\left(u_{1}, u_{2}\right) \in F$ and let $x_{u} \in[0,1]$. Let us divide the proof of (20) into two natural cases:

1. $x_{u} \notin(\alpha, \beta)$. Then $\xi\left(x_{u}\right)=\varphi\left(x_{u}\right)$ and, consequently,

$$
\varrho\left(\left(u_{1}, u_{2}\right),\left(x_{u}, \xi\left(x_{u}\right)\right)\right) \geq \tau_{\varphi} \geq \sigma_{0} .
$$

2. $x_{u} \in[\alpha, \beta]$. Then, according to (18), $\xi\left(x_{u}\right) \in\left[\varphi\left(x_{0}\right)-\frac{\sigma}{2}, \varphi\left(x_{0}\right)-\frac{\sigma}{2}\right]$. According to (16) and the assumption $\sigma \in(0, \varepsilon]$ we have

$$
\varrho\left(\left(u_{1}, u_{2}\right),\left(x_{u}, \xi\left(x_{u}\right)\right)\right) \geq \frac{\sigma}{4} \geq \sigma_{0} .
$$

Consequently, $\varrho\left(\left(u_{1}, u_{2}\right), \xi\right) \geq \sigma_{0}$, which proves (20).

Now, let us fix $\eta_{0} \in V$. We will prove

Let $x_{*} \in[0,1]$. Then,

$$
\eta_{0} \cap F=\emptyset \text {. }
$$

$$
\varrho\left(\left(x_{*}, \eta_{0}\left(x_{*}\right)\right), \xi\right) \leq \varrho\left(\left(x_{*}, \eta_{0}\left(x_{*}\right)\right),\left(x_{*}, \xi\left(x_{*}\right)\right)\right)<\sigma_{0} .
$$

If we combine this inequality with (20), we obtain $\left(x_{*}, \eta_{0}\left(x_{*}\right)\right) \notin F$ and, according to arbitrariness of the choice of $x_{*} \in[0,1]$, we have (21), and consequently the proof of (19) is finished.

From (18) and the definition of $\xi$, it follows that

$$
\eta(x) \in\left[\varphi\left(x_{0}\right)-\sigma, \varphi\left(x_{0}\right)+\sigma\right], \quad \text { for } \quad \eta \in V \text { and } x \in[\alpha, \beta] .
$$

Consequently, in the case $2^{\circ}$, taking (17) into account, we conclude that there is no point belonging to $[\alpha, \beta] \cap \operatorname{Fix}(\eta)$. The proof of this theorem with respect to the case $2^{\circ}$ is finished. 
From now on, we will consider only the first case. Repeating ideas of considerations proving (9) in the proof of Theorem 3.3, we may infer that each point belonging to $(\alpha, \beta)$ is $\infty$-entropy point of $\eta$ and, consequently,

$$
z \notin \mathrm{E}_{0}(\eta), \quad \text { for any } \quad z \in(\alpha, \beta) \text {. }
$$

Then, $x_{0}$ is not an extremely chaotic point of $\eta$ also in the case $1^{\circ}$. The arbitrariness of the choice of $\eta \in V$ and our assumptions that $\mathfrak{F}$ is a family having extremely chaotic property at $x_{0}$ led us to the conclusion that

$$
V \cap \mathfrak{F}=\emptyset \text {. }
$$

\section{REFERENCES}

[1] JASTRZĘBSKI, J. M.-JĘDRZEJEWSKI, J. M.-NATKANIEC, T.: On some subclass of Darboux functions, Fund. Math. 138 (1991), 165-173.

[2] KELLY, J. C.: Bitopological spaces, Proc. London Math. Soc. (3) 13 (1963), 71-89.

[3] KORCZAK-KUBIAK, E.-LORANTY, A.-PAWLAK, R. J.: On $\infty$-entropy points in real analysis, Opuscula Math. (to appear).

[4] KWIETNIAK, D.-OPROCHA, P.: Topological entropy and chaos for maps induced on hyperspaces, Chaos Solitons Fractals 33 (2007), 76-86.

[5] LORANTY, A.-PAWLAK, R. J.: The generalized entropy in the generalized topological spaces, Topol. Appl. 159 (2012), 1734-1742.

[6] NATKANIEC, T.: Almost Continuity, Habilitation Thesis, Bydgoszcz, 1992.

[7] NATKANIEC, T.: Almost continuity, Real Anal. Exchange 17 (1991-92), 462-520.

[8] NITECKI, Z. H.: Topological entropy and the preimage structure of maps, Real Anal. Exchange 29 (2003/2004), 9-42.

[9] NOWIK, A.: Notes on the ideal (a), Tatra Mt. Math. Publ. 46 (2010), 41-45.

[10] PAWLAK, H.-PAWLAK, R. J.: On $T_{\Gamma}$-approximation of functions by means of derivatives and approximately continuous functions having local periodic property, in: Real Functions, Density Topology and Related Topics (M. Filipczak et al., eds.), Łódź University Press, Łódź, 2011, pp. 101-111.

[11] PAWLAK, R. J.-LORANTY, A.-BAKOWSKA, A.: On the topological entropy of continuous and almost continuous functions, Topology Appl. 158 (2011), 2022-2033.

[12] PAWLAK, R. J.: On the entropy of Darboux functions, Colloq. Math. 116 (2009), 227-241.

[13] STALlings, J.: Fixed point theorem for connectivity maps. Fund. Math. 47 (1959), 249-263.

College of Computer Science

University of Eódź

Banacha 22

$P L-90-238$ Eódź

POLAND

E-mail: rpawlak@math.uni.lodz.pl 